\title{
AVERAGED INERTIA PRINCIPLE FOR NON-NEWTONIAN SQUEEZE FILMS IN WIDE PARALLEL PLATES: COUPLE STRESS FLUID
}

\author{
Jaw-Ren Lin \\ Professor, Department of Mechanical Engineering, Nanya Institute of Technology, P.O. Box 324-22-59, Jhongli, Taiwan \\ 320, R.O.C., jrlin@nanya.edu.tw \\ Chi-Ren Hung \\ Instructor, Department of Mechanical Engineering, Nanya Institute of Technology, P.O. Box 324-22-59, Jhongli, Taiwan \\ 320, R.O.C. \\ Rong-Fang Lu \\ Assistant Professor, Department of Mechanical Engineering, Nanya Institute of Technology, P.O. Box 324-22-59, \\ Jhongli, Taiwan 320, R.O.C.
}

Follow this and additional works at: https://jmstt.ntou.edu.tw/journal

Part of the Mechanical Engineering Commons

\author{
Recommended Citation \\ Lin, Jaw-Ren; Hung, Chi-Ren; and Lu, Rong-Fang (2006) "AVERAGED INERTIA PRINCIPLE FOR NON-NEWTONIAN \\ SQUEEZE FILMS IN WIDE PARALLEL PLATES: COUPLE STRESS FLUID," Journal of Marine Science and Technology. \\ Vol. 14: Iss. 4, Article 13. \\ DOI: $10.51400 / 2709-6998.2092$ \\ Available at: https://jmstt.ntou.edu.tw/journal/vol14/iss4/13 \\ This Research Article is brought to you for free and open access by Journal of Marine Science and Technology. It has been \\ accepted for inclusion in Journal of Marine Science and Technology by an authorized editor of Journal of Marine Science and \\ Technology.
}


AVERAGED INERTIA PRINCIPLE FOR NON-NEWTONIAN SQUEEZE FILMS IN WIDE PARALLEL PLATES: COUPLE STRESS FLUID

Acknowledgements

The authors would like to thank the National Science Council of ROC for the support through Grant: NSC-94-2212-E-253-005-. 


\title{
AVERAGED INERTIA PRINCIPLE FOR NON-NEWTONIAN SQUEEZE FILMS IN WIDE PARALLEL PLATES: COUPLE STRESS FLUID
}

\author{
Jaw-Ren Lin*, Chi-Ren Hung**, and Rong-Fang Lu***
}

Key words: convective inertia forces, couple-stress fluids, squeezing films, parallel plates, micro-continuum theory.

\section{ABSTRACT}

On the basis of the Stokes micro-continuum theory, the combined effects of non-Newtonian couple stresses and convective fluid inertia forces upon the squeeze film characteristics of two wide parallel plates are investigated by using the averaged inertia principle. A numerical solution of the film height versus response time is calculated by using the fourth-order Runge-Kutta method. Compared to the Newtonian-lubricant non-inertia case of Hamrock [4], the qualitative effects of couple stresses and convective inertia forces provide an increase in the film pressure, the load capacity and the response time. Moreover, the quantitative effects are more pronounced for wide parallel plates operating at a smaller squeeze film height, as well as a larger couple stress parameter and film Reynolds number. The results of the present investigation provide useful information for engineers to design a parallel-plate squeeze-film system considering both the effects of convective inertia forces and non-Newtonian couple stresses.

\section{INTRODUCTION}

Research of squeeze film behaviors plays an important role in many engineering applications, such as, for example, turbo-machinery, matching gears, viscous dampers, hydraulic actuators, braking units and skeletal joints. Traditionally, study of squeeze film characteristics focuses upon the use of Newtonian lubricants for various types of film surfaces by Hamrock [4], Hays [5], Gupta and Vora [3] and Murti [10]. Owing to the development modern machine apparatuses, the increas-

Paper Submitted 12/27/05, Accepted 03/27/06. Author for Correspondence: Jaw-Ren Lin. E-mail: jrlin@nanya.edu.tw.

*Professor, Department of Mechanical Engineering, Nanya Institute of Technology, P.O. Box 324-22-59, Jhongli, Taiwan 320, R.O.C.

**Instructor, Department of Mechanical Engineering, Nanya Institute of Technology, P.O. Box 324-22-59, Jhongli, Taiwan 320, R.O.C.

***Assistant Professor, Department of Mechanical Engineering, Nanya Institute of Technology, P.O. Box 324-22-59, Jhongli, Taiwan 320, R.O.C. ing use of fluids containing a microstructure such as those containing additives, suspensions, granular matter or long-chained polymers has been emphasized. To explain the peculiar behaviors of these kinds of nonNewtonian fluids, the micro-continuum theory proposed by Stokes [13] is the simplest generalization of the classical theory of fluids which allows for polar effects such as the presence of an anti-symmetric stress tensor, couple stresses and body couples. It is intended to account for the particle size effects of additives. The couple stress effects are considered as a consequence of the action of a deforming body on its neighborhood. The equations of motion for a Stokes incompressible fluid with couple stresses are

$$
\begin{aligned}
\rho\left(\frac{\partial \vec{V}}{\partial t}+\vec{V} \cdot \nabla \vec{V}\right) & =-\nabla p+\rho \vec{B}+\frac{1}{2} \rho \nabla \times \vec{C}+\mu \nabla^{2} \vec{V} \\
& -\eta \nabla^{4} \vec{V}
\end{aligned}
$$

where the vectors $\vec{V}, \vec{B}$ and $\vec{C}$ represent respectively the velocity, the body force per unit mass and the body couple per unit mass, $\rho$ is the density, $p$ is the pressure, $\mu$ is the viscosity coefficient, and $\eta$ is a new material constant with the dimension of momentum. Many investigators have applied this theory of couple stress fluids to study the squeeze film characteristics of different systems, such as the squeeze film plates of various shapes by Ramanaiah [12], the squeeze film configuration with reference to synovial joints by Bujurke and Jayaraman [1] and Lin [6], the squeeze film partial journal bearings by Lin [7], the squeeze film sphereplate mechanism by Lin [8] and the squeeze film cylinder-surface system by Lin et al. [9]. However, all the above studies neglected the effects of fluid inertia forces as compared to the viscous forces. Since the effects of squeezing film pressure becomes more and more significant as the fluid velocity increases, the squeeze film characteristics taking into account the convective fluid inertia forces should be included. 
On the ground of the micro-continuum theory, the combined effects of non-Newtonian couple stresses and convective fluid inertia forces on the performance characteristics in two parallel squeeze-film plates are concerned. By applying the averaged inertia principle, the lubrication equation is derived from Stokes motion equations to account for the couple stress effects resulting from the lubricant blended with various types of additives. The traditional results for the Newtonian non-inertia flow by Hamrock [4] are used as a particular comparison. To reveal the combined effects of fluid convective inertia forces and non-Newtonian couple stresses, the performance characteristics of squeeze film parallel plates will be presented under different values of the Reynolds number and the couple stress parameter.

\section{ANALYSIS}

According to the Stokes micro-continuum theory, the continuity equation and the motion equations governing the motion of an incompressible couple stress fluid in the absence of body forces and body couples give

$$
\begin{aligned}
& \nabla \cdot \vec{V}=0 \\
& \rho\left(\frac{\partial \vec{V}}{\partial t}+\vec{V} \cdot \nabla \vec{V}\right)=-\nabla p+\mu \nabla^{2} \vec{V}-\eta \nabla^{4} \vec{V}
\end{aligned}
$$

For the present study Figure 1 describes the squeeze film geometry of two parallel plates of length $L$ with width $B \gg L$, in which the upper surface is approaching the lower one with a squeezing velocity $V=-d h / d t$. Assume the thin-film theory of hydrodynamic lubrication is applicable, but the effects of fluid convective inertia forces due to temporal acceleration of the fluid are considered. Then equations of continuity and mo-

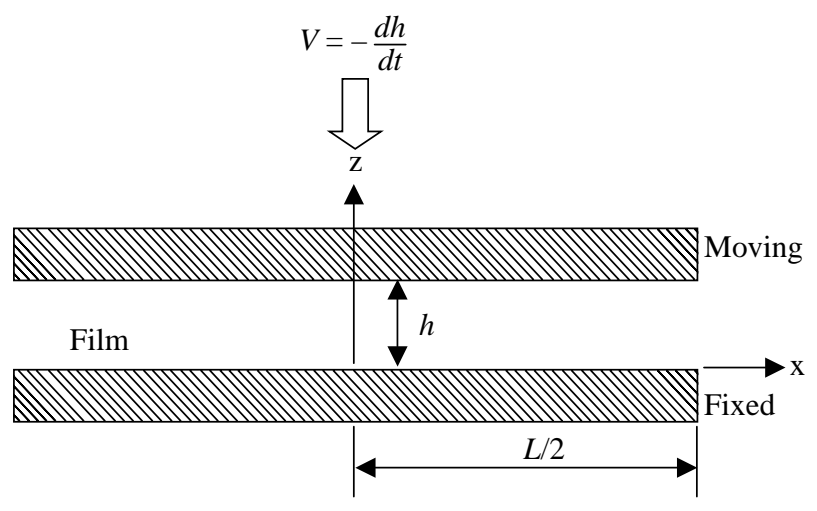

Fig. 1. Squeeze film geometry between two wide parallel plates. tion can be expressed as

$$
\begin{aligned}
& \frac{\partial u}{\partial x}+\frac{\partial w}{\partial z}=0 \\
& \rho\left(u \frac{\partial u}{\partial x}+w \frac{\partial u}{\partial z}\right)=-\frac{\partial p}{\partial x}+\mu \frac{\partial^{2} u}{\partial z^{2}}-\eta \frac{\partial^{4} u}{\partial z^{4}} \\
& \frac{\partial p}{\partial z}=0
\end{aligned}
$$

where $u$ and $w$ represent the velocity components in the $x$ and $z$ directions respectively. The boundary conditions for the velocity components $u$ and $w$ at the plate/film surfaces are

$$
\begin{aligned}
& u=0, \quad \frac{\partial^{2} u}{\partial z^{2}}=0, \quad w=0 \quad \text { at } \quad z=0 \\
& u=0, \quad \frac{\partial^{2} u}{\partial z^{2}}=0, \quad w=\frac{\partial h}{\partial t} \quad \text { at } \quad z=h
\end{aligned}
$$

By the analysis of the order of magnitude by Pinkus and Sternlicht [11], since the film thickness $h$ is small, the convective inertia forces in Eq. (5) can be treated by the mean value averaged across the film thickness in the following manner:

$$
\frac{\rho}{h} \int_{z=0}^{h}\left(u \frac{\partial u}{\partial x}+w \frac{\partial u}{\partial z}\right) d z=-\frac{\partial p}{\partial x}+\mu \frac{\partial^{2} u}{\partial z^{2}}-\eta \frac{\partial^{4} u}{\partial z^{4}}
$$

The left-hand side of the above equation after integration yields the function, $(\rho / h) d\left(\int_{z=0}^{h} u^{2} d z\right) / d x$. By introducing the modified pressure gradient $g_{m p}$,

$$
g_{m p}=\frac{\partial p}{\partial x}+\frac{\rho}{h}\left(\frac{d}{d x} \int_{z=0}^{h} u^{2} d z\right)
$$

and solving Eq. (9) together with continuity Eq. (4) and boundary conditions (7) and (8), one can obtain

$$
u=\frac{1}{2 \mu} g_{m p}\left\{z^{2}-h z+2 l^{2}-2 l^{2} \frac{\cosh [(2 z-h) / 2 l]}{\cosh (h / 2 l)}\right\}
$$

where $l$ is defined by

$$
l=(\eta / \mu)^{1 / 2}
$$

The ratio of $\eta / \mu$ has dimension of length squared and, therefore, $l$ characterizes the material length of fluid. Substitute the expression of $u$ into the equation of squeezing motion 


$$
\frac{d}{d x} \int_{z=0}^{h} u d z+\frac{d h}{d t}=0
$$

Performing the integration and applying a symmetrical condition at $x=0$, one can obtain the modified pressure gradient function,

$$
g_{m p}=12 \mu \frac{x}{h^{3}-12 l^{2} h+24 l^{3} \tan (h / 2 l)} \frac{d h}{d t}
$$

Once the modified pressure gradient function has been derived, the squeeze film pressure, the load-carrying capacity and the time-height relationship can be evaluated.

\section{SQUEEZE FILM CHARACTERISTICS}

Substituting the modified pressure gradient $g_{m p}$ from (14) and the velocity component $u$ from (11) into Eq. (10), one can derive the lubrication equation is derived as

$$
\frac{\partial p}{\partial x}=\frac{12 \mu x}{f_{0}(h, l)} \frac{d h}{d t}-\frac{72 \rho x f_{1}(h, l)}{h f_{0}^{2}(h, l)}\left(\frac{d h}{d t}\right)^{2}
$$

The functions $f_{0}(h, l)$ and $f_{1}(h, l)$ are defined by

$$
\begin{aligned}
f_{0}(h, l) & =h^{3}-12 l^{2} h+24 l^{3} \tanh \left(\frac{h}{2 l}\right) \\
f_{1}(h, l) & =\frac{h^{5}}{30}-\frac{2}{3} l^{2} h^{3}+\left[14-2 \tanh ^{2}\left(\frac{h}{2 l}\right)\right] l^{4} h \\
& -28 l^{5} \tanh \left(\frac{h}{2 l}\right)
\end{aligned}
$$

The boundary conditions for the film pressure are

$p=0 \quad$ at $\quad x= \pm L / 2$

Integrating once with respect to $x$ with the above conditions gives the film pressure,

$$
p=\left[\frac{12 \mu}{f_{0}(h, l)} \frac{d h}{d t}-\frac{72 \rho f_{1}(h, l)}{h f_{0}^{2}(h, l)}\left(\frac{d h}{d t}\right)^{2}\right]\left(\frac{x^{2}}{2}-\frac{L^{2}}{8}\right)
$$

Expressing in a non-dimensional form, one can achieve

$$
p^{*}=\left[\frac{6}{f_{0}^{*}\left(h^{*}, l^{*}\right)}+\frac{36 \operatorname{Re} f_{1}^{*}\left(h^{*}, l^{*}\right)}{h^{*} f_{0}^{* 2}\left(h^{*}, l^{*}\right)}\right]\left(\frac{1}{4}-x^{* 2}\right)
$$

The dimensionless variables and parameters in the above equation are defined as

$$
x^{*}=\frac{x}{L}, \quad h^{*}=\frac{h}{h_{0}}, \quad l^{*}=\frac{l}{h_{0}} \quad p^{*}=\frac{p h_{0}^{3}}{\mu L^{2}(-d h / d t)}
$$

$$
\begin{aligned}
& R_{e}=\frac{\rho h_{0}(-d h / d t)}{\mu}, f_{0}^{*}\left(h^{*}, l^{*}\right)=\frac{f_{0}(h, l)}{h_{0}^{3}}, \\
& f_{1}^{*}\left(h^{*}, l^{*}\right)=\frac{f_{1}(h, l)}{h_{0}^{5}}
\end{aligned}
$$

where $h_{0}$ is the initial film thickness, $l^{*}$ denotes the couple stress parameter, and $R_{e}$ represents the Reynolds number.

The load-carrying capacity per unit width is now obtained by integrating the film pressure acting upon the upper plate,

$$
\frac{W}{B}=\int_{x=-L / 2}^{L / 2} p d x
$$

After performing the integration, the dimensionless load-carrying capacity is obtained,

$$
\begin{aligned}
W^{*} & =\frac{W h_{0}^{3}}{\mu L^{3} B(-d h / d t)}=\frac{1}{f_{0}^{*}\left(h^{*}, l^{*}\right)} \\
& +6 R_{e} \frac{f_{1}^{*}\left(h^{*}, l^{*}\right)}{h^{*} f_{0}^{* 2}\left(h^{*}, l^{*}\right)}
\end{aligned}
$$

As the value of $R_{e}$ is equal to zero, the dimensionless load-carrying capacity $W^{*}$ reduces to the non-inertia load capacity of the parallel plates with couple stress fluids,

$$
W^{*}=\frac{1}{f_{0}^{*}\left(h^{*}, l^{*}\right)}
$$

In addition, as both the values of $R_{e}$ and $l^{*}$ approach zero, the dimensionless load-carrying capacity agrees well with the non-inertial Newtonian-lubricant case of Hamrock [4],

$$
W^{*}=\frac{1}{h^{* 3}}
$$

Now, we introduce the dimensionless response time,

$$
t^{*}=\frac{W h_{0}^{2}}{\mu L^{3} B} t
$$

The time-height relationship is then obtained from 
Eq. (24) as

$$
\frac{d h^{*}}{d t^{*}}=-\frac{h^{*} f_{0}^{* 2}\left(h^{*}, l^{*}\right)}{h^{*} f_{0}^{*}\left(h^{*}, l^{*}\right)+6 R_{e} f_{1}^{*}\left(h^{*}, l^{*}\right)}
$$

The equation of time-height relationship (18) is observed to be a highly nonlinear ordinary differential equation. The initial condition for the dimensionless film thickness is given by

$$
h^{*}=1 \quad \text { at } \quad t^{*}=0
$$

A numerical solution of the film height versus response time can be evaluated by using a fourth-order Runge-Kutta method. The first-order Runge-Kutta method approaches the solution with a series of short straight-line segments; it may yield inaccurate results when the slope of the solution varies rapidly or if the step size is large. The higher-order Runge-Kutta method averages several tangent-line approximations to the solution. One can use a subroutine ode 45 of the fourthorder Runge-Kutta method designed in the commercial mathematical language, named Matlab. The default tolerance related to the step size is 0.000001 . Therefore a steadily convergent result can be obtained. More details about the fourth-order Runge-Kutta method and the use of ode45 in Matlab can be found by Etter [2].

\section{RESULTS AND DISCUSSION}

Applying the Stokes micro-continuum theory and
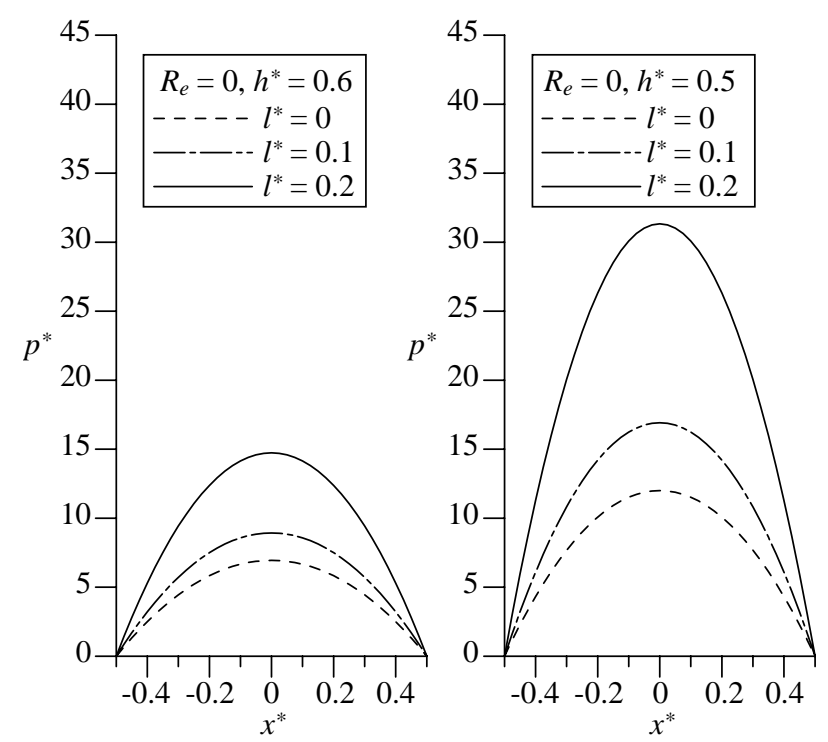

Fig. 2. Variation of film pressure $p^{*}$ with coordinate $x^{*}$ for different $h^{*}$ and $l^{*}$ under the non-inertia case $\boldsymbol{R}_{e}=0$. the averaged inertia principle, the combined effects of non-Newtonian couple stresses and convective fluid inertia forces upon the squeeze film performance of two parallel plates are investigated. From the aid of the definition in Eqs. (21) and (12), the couple stress parameter $l^{*}$ dominates the couple stresses effects resulting from a lubricant blended with various types of additives. With the aid of the definition in Eq. (22), the film Reynolds number $R_{e}$ signifies the convective inertia force effects resulting from temporal acceleration of the fluid. For both the values of $R_{e}$ and $l^{*}$ approaching zero, the results for the non-inertia Newtonian-lubricant case of Hamrock [4] are recovered.

Film pressure. Figure 2 displays the variation of dimensionless film pressure $p^{*}$ with dimensionless coordinate $x^{*}$ for different values of film height $h^{*}$ and couple stress parameter $l^{*}$ under the non-inertia case $R_{e}=0$. Comparing with the Newtonian-lubricant case $\left(l^{*}=0\right)$ at film height $h^{*}=0.6$, the effects of couple stresses $\left(l^{*}=0.1,0.2\right)$ result in a higher film pressure. In addition, the lower the squeeze film height achieves $\left(h^{*}\right.$ $=0.5)$, the more the couple stresses affect the film pressure. Figure 3 presents the variation of dimensionless film pressure $p^{*}$ with dimensionless coordinate $x^{*}$ for different values of Reynolds number $R_{e}$ with couple stress parameter $l^{*}=0$ and 0.1 . The effects of convective inertia $\left(R_{e}=5\right)$ signify an increase in the value of squeeze film pressure under both of the Newtonianlubricant case $\left(l^{*}=0\right)$ and non-Newtonian-lubricant case $\left(l^{*}=0.1\right)$. Further increments of $p^{*}$ are obtained for a higher Reynolds number $\left(R_{e}=10\right)$.

Load-carrying capacity. Figure 4 shows the varia-
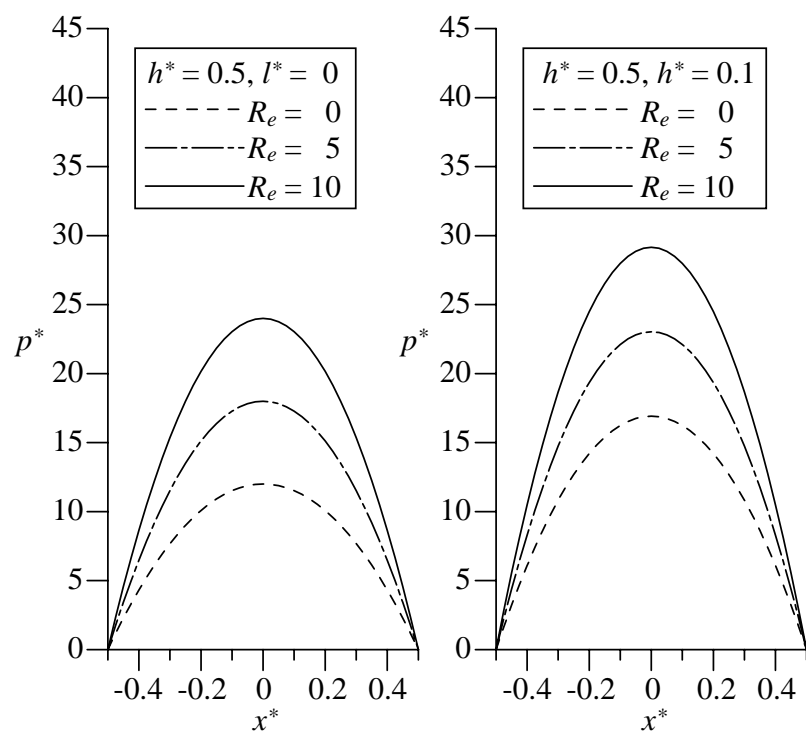

Fig. 3. Variation of film pressure $p^{*}$ with coordinate $x^{*}$ for different $R_{e}$ with $l^{*}=0$ and 0.1 . 
tion of dimensionless load-carrying capacity $W^{*}$ with couple stress parameter $l^{*}$ for different values of film height $h^{*}$ under the non-inertia case $R_{e}=0$. Since the couple stress effects yield a higher film pressure, the integrated load-carrying capacity is similarly affected. Comparing with the Newtonian-lubricant case $\left(l^{*}=0\right)$, the effects of couple stresses increase the load-carrying capacity; and larger increments are obtained with decreasing value of $h^{*}$ or increasing value of $l^{*}$. Figure 5 describes the variation of dimensionless load-carrying capacity $W^{*}$ with Reynolds number $R_{e}$ for different values of film height $h^{*}$ under both the Newtonianlubricant $l^{*}=0$ and non-Newtonian-lubricant $l^{*}=0.1$ cases. The load capacity is observed to increase with increasing values of the Reynolds number. On average, the combined effects of convective fluid inertia and couple stresses on the load-carrying capacity are emphasized at a lower film height.

Response time. Figure 6 presents the variation of dimensionless film height $h^{*}$ with dimensionless response time $t^{*}$ for different values of couple stress parameter $l^{*}$ under the non-inertial case $R_{e}=0$. It is observed that the presence of couple stresses provides an increase in the response time. These phenomena can be realized that since the effects of non-Newtonian couple stresses result in a higher load-carrying capacity, a higher film thickness would be attained for the same time to be taken as compared to the Newtonian-lubricant case. Figure 7 shows the variation of film height $h^{*}$ with response time $t^{*}$ for different Reynolds numbers

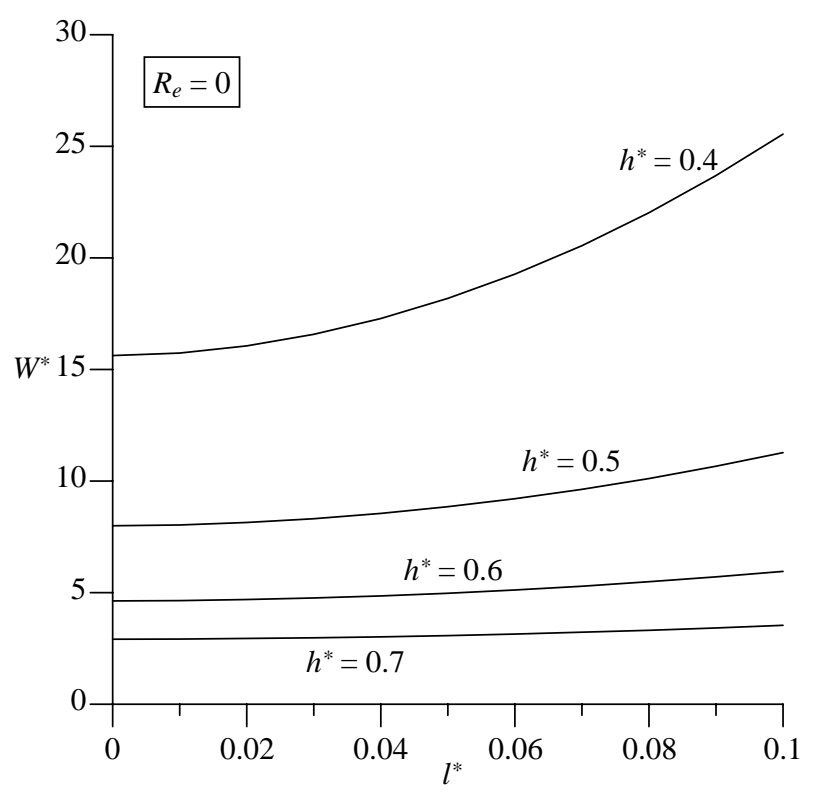

Fig. 4. Variation of load capacity $W^{*}$ with couple stress parameter $l^{*}$ for different $\boldsymbol{h}^{*}$ under the non-inertia case $\boldsymbol{R}_{e}=\mathbf{0}$.
$R_{e}$ under both the Newtonian-lubricant and nonNewtonian-lubricant cases. Since the effects of convective fluid inertia forces result in a higher loadcarrying capacity as described in Figure 5, a longer response time is observed for the squeeze film plates as compared to the non-inertial case. Further increments of response time are emphasized by the use of a couple stress fluid $\left(l^{*}=0.1\right)$.
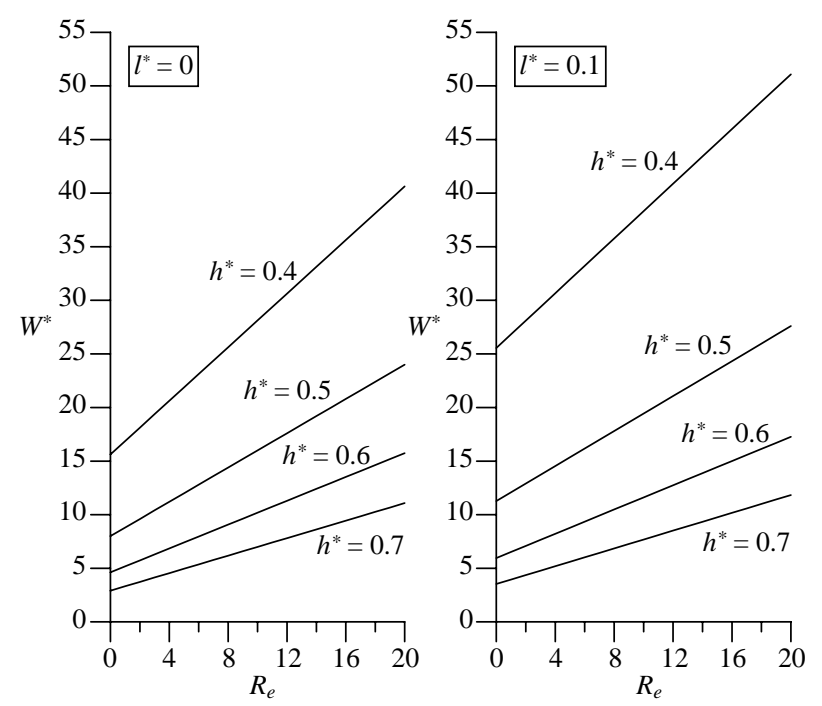

Fig. 5. Variation of load capacity $W^{*}$ with Reynolds number $\boldsymbol{R}_{\mathrm{e}}$ for different $h^{*}$ under both the Newtonian-lubricant and nonNewtonian-lubricant cases.

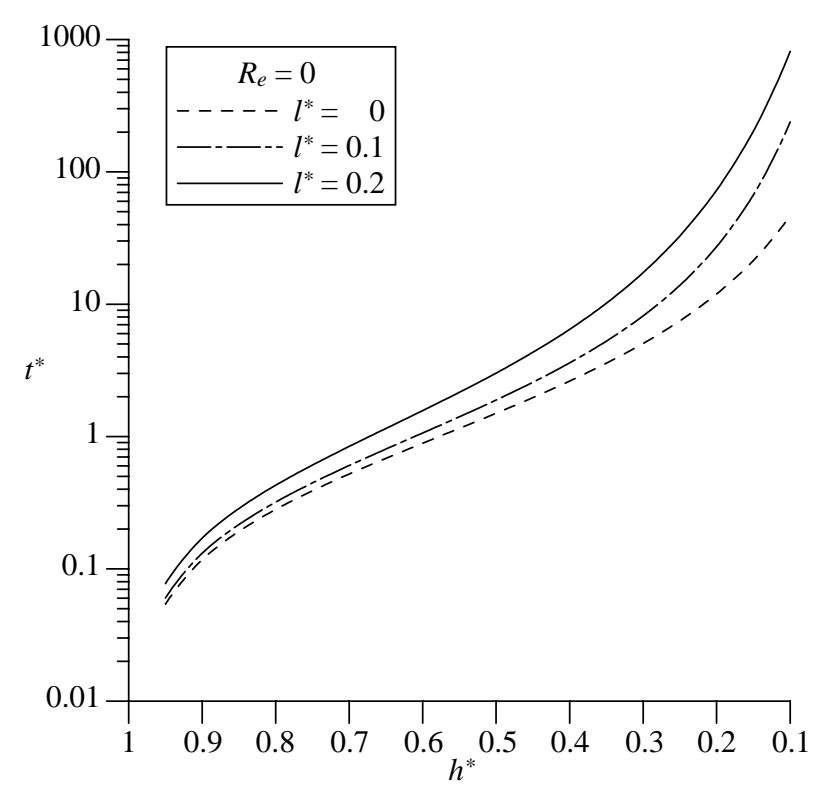

Fig. 6. Variation of film height $h^{*}$ with response time $t^{*}$ for different $h^{*}$ under the non-inertia case $\boldsymbol{R}_{e}=\mathbf{0}$. 

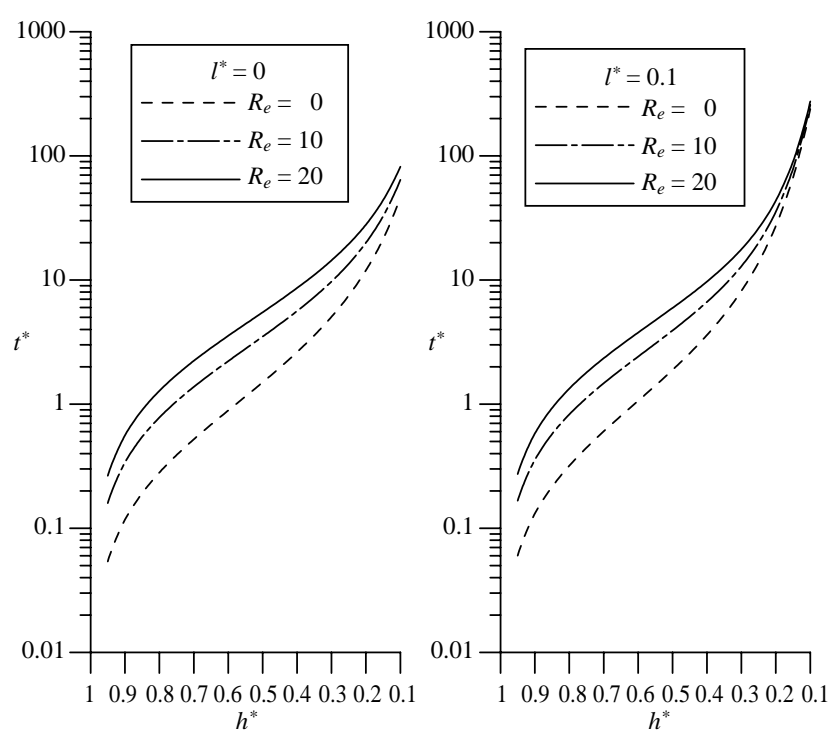

Fig. 7. Variation of film height $h^{*}$ with response time $t^{*}$ for different $R_{\mathrm{e}}$ under both the Newtonian-lubricant and non-Newtonian-lubricant cases.

Combined effects. In order to gain an insight into the height-time relationship, the variation of dimensionless film height $h^{*}$ with dimensionless response time $t^{*}$ for different values of couple stress parameter $l^{*}$ and Reynolds number $R_{e}$ is presented in Figure 8. By the use of a non-Newtonian couple stress fluid $\left(l^{*}=0.1, R_{e}\right.$ $=0$ ), the squeezing plates result in a longer response time as compared to the Newtonian-lubricant non-inertia case $\left(l^{*}=0, R_{e}=0\right)$. Considering the temporal acceleration of the fluid $\left(l^{*}=0.1, R_{e}=10\right)$, the effects of convective fluid inertia provide a visible increase in the response time. Increasing both the values of couple stress parameter $\left(l^{*}=0.2, R_{e}=10\right)$ and Reynolds number $\left(l^{*}=0.2, R_{e}=20\right)$, further increments of the response time are predicted for the squeezing film plates. Totally, the combined effects of couple stresses and convective inertia forces upon the squeeze film behavior between parallel plates are apparent. The response times for the squeeze film plates are significantly lengthened by the use of an incompressible couple stress fluid and considering the convective inertia force effects.

\section{CONCLUSIONS}

On the ground of the Stokes micro-continuum theory and the principle of averaged inertia, the combined effects of non-Newtonian couple stresses and convective fluid inertia forces upon the squeeze film behavior of two wide parallel squeezing plates are presented. The squeeze-film film pressure and the loadcarrying capacity are calculated. A fourth-order Runge-

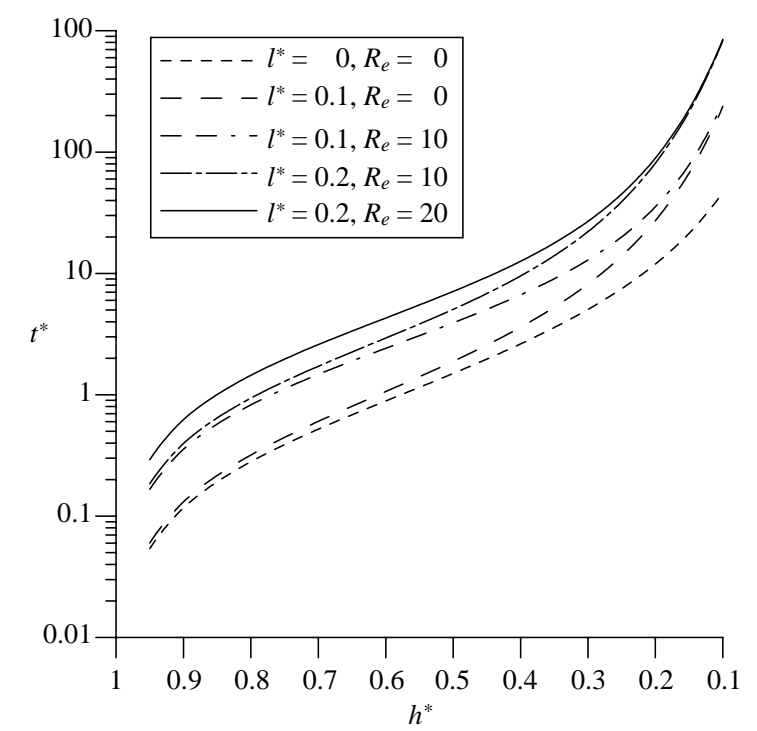

Fig. 8. Variation of film height $h^{*}$ with response time $t^{*}$ for different values of $l^{*}$ and $\boldsymbol{R}_{\boldsymbol{e}}$.

Kutta method has been applied to evaluate the film height versus response time. In accordance with the results and discussion, conclusions are drawn as follows.

1. Considering both the effects of non-Newtonian couple stresses and convective inertia forces, we have derived a closed-form solution for the film pressure between wide parallel squeeze-film plates. It is found that there are two parameters dominating the combined effects in the squeezing film motion: the couple stress parameter $l^{*}$ signifying the non-Newtonian couple stress effects resulting from a lubricant blended with various types of additives, and the film Reynolds number $R_{e}$ characterizing the convective inertia force effects arising from the temporal acceleration of fluids.

2. Comparing with the Newtonian-lubricant non-inertia case, the effects of couple stresses increase the film pressure especially for larger couple stress parameters. More increments are emphasized when the squeeze film height is small and/or when the effects of convective inertia are further considered.

3. Comparing with the Newtonian-lubricant non-inertia case, the combined effects of couple stresses and convective inertia forces provide an enhancement in the load-carrying capacity as well as an increase in the response time of the squeeze film behavior.

4. The quantitative effects of couple stresses and convective inertia forces on the improved squeeze-film characteristics are more pronounced with a smaller squeeze film height, a larger couple stress parameter and a larger film Reynolds number. 


\section{ACKNOWLEDGEMENT}

The authors would like to thank the National Science Council of ROC for the support through Grant: NSC-94-2212-E-253-005-.

\section{REFERENCES}

1. Bujurke, N.M. and Jayaraman, G., "The Influence of Couple Stresses in Squeeze Films," International Journal of Mechanical Sciences, Vol. 24, pp. 369-376 (1982).

2. Etter, D.M., Engineering Problem Solving with Matlab, Prentice Hall, London, pp. 205-212 (1997).

3. Gupta, J.L. and Vora, K.H., "Analysis of Squeeze Films between Curved Annular Plates," Journal of Lubrication Technology, Vol. 102, pp. 48-50 (1980).

4. Hamrock, B.J., Fundamental of Fluid Film Lubrication, McGraw-Hill, New York, pp. 279-283 (1994).

5. Hays, D.F., "Squeeze Films for Rectangular Plates," Journal of Basic Engineering, Vol. 88, pp. 243-246 (1963).

6. Lin, J.R., "Couple-stress Effects on the Squeeze Film Characteristics of Hemispherical Bearings with Reference to Synovial Joints," International Journal of Me- chanics and Engineering, Vol. 1, pp. 317-332 (1996).

7. Lin, J.R., "Squeeze Film Characteristics of Finite Journal Bearings: Couple Stress Fluid Model," Tribology International, Vol. 31, pp. 201-207 (1998).

8. Lin, J.R., "Squeeze Film Characteristics between a Sphere and a Flat Plate: Couple Stress Fluid Model," Computers and Structures, Vol. 75, pp. 73-80 (2000).

9. Lin, J.R., Liao, W.H., and Hung, C.R., "The Effects of Couple Stresses in the Squeeze Film Characteristics between a Cylinder and a Plane Surface," Journal of Marine Science and Technology, Vol. 12, pp. 119-123 (2004).

10. Murti, P.R.K., "Squeeze Films in Curved Circular Plates," Journal of Lubrication Technology, Vol. 79, pp. 650-652 (1975).

11. Pinkus, O. and Sternlicht, B., Theory of Hydrodynamic Lubrication, McGraw-Hill, New York, pp. 352-361 (1961).

12. Ramanaiah, G., "Squeeze Films between Finite Plates Lubricated by Fluids with Couple Stress," Wear, Vol. 54, pp. 315-320 (1979).

13. Stokes, V.K., "Couple Stresses in Fluids," The Physics of Fluids, Vol. 9, pp. 1709-1715 (1966). 